\title{
Kant Y La CoMunicación de IdeAs Estéticas
}

\section{Kant and the Aesthetic Ideas' Communication}

\author{
Adryan Fabrizio Pineda Repizzo* \\ Universidad Autónoma de Colombia \\ faospace@gmail.com
}

Fecha de recepción: 13 de marzo de 2014

Fecha de aceptación: 29 de mayo de 2014

\section{Resumen}

Este artículo de reflexión explora el planteamiento kantiano en torno a la comunicación de ideas estéticas; su hipótesis plantea que las características de la estética de Kant y el papel que este otorga a las ideas estéticas pueden dar lugar a la reflexión conceptual en torno al rol que ocupan hoy en el arte los conceptos y la expectativa de recepción del observador. El análisis planteado busca destacar la pertinencia de las relaciones entre forma e idea estética para un análisis filosófico del arte.

Palabras clave: Estética, Idea estética, Kant.

\begin{abstract}
This reflection paper explores the Kantian approach about the aesthetic ideas' communication. The hypothesis is that the Kant aesthetics' features, and the role he assigns to the aesthetic ideas, can lead to the conceptual reflection, on the role that concepts occupies today in art, and their receipt expectation by the observer. Thus, the proposed analysis seeks to highlight the relationships between the aesthetic form and idea's relevance, for an art philosophical analysis.
\end{abstract}

Keywords: Aesthetic, Aesthetic Idea, Judgement, Kant.

* Magíster en Filosofía, de la Universidad del Rosario. Magíster en Estudios Culturales, de la Universidad de los Andes. Docente investigador de la Facultad de Ciencias Humanas de la Universidad Autónoma de Colombia. faospace@gmail.com 
El artista colombiano Bernardo Salcedo fue uno de los pioneros del arte conceptual en Colombia, el cual, en términos generales, se caracteriza por hacer del objeto artístico la expresión de un concepto tal que, en sentido estricto, es la obra de arte expuesta. He ahí el problema que se debe comprender: ¿cómo puede un concepto ser una obra de arte?, ¿cómo podemos entender algo así, como un concepto artístico? Consideremos el caso de un trabajo de este artista. Hectáreas de heno fue una obra producida en el año 2000, presentaba un grupo numeroso de bolsas llenas de heno, cada una marcada con distintas magnitudes, en el medio de la sala de exposición. De esta obra poco podemos decir acerca de unas "cualidades" o "propiedades" de la forma y la materia que nos lleven a comprender que tenemos en frente de nosotros una obra de arte. Probablemente asumiríamos que por el hecho de estar en una sala museal es ya una obra de arte, además del reconocimiento social del artista y cualquier otro factor social. Sin embargo, cabe preguntarnos: ¿queda aún algún tipo de experiencia singular frente a esta obra? El arte conceptual espera que el espectador sea confrontado por el concepto que la obra manifiesta. ¿Cómo es posible mantener esta expectativa? La idea que asumiremos a continuación es que la estética kantiana y, en particular, la noción de "idea estética", pueden proveer unas herramientas para dilucidar la posibilidad de afirmar este singular tipo de comunicación estética ${ }^{1}$. Además, la estética kantiana nos pone de frente algo que el arte conceptual plasma: es un tipo de expresión de las ideas que no es el mismo de la ciencia o de la técnica, sino que es propio del arte, es una manera de expresión de las ideas que solo puede hacer el artista o, en palabras de Gadamer, "solo en el artista ocurre que su invento, su obra, mantiene en su ser una referencia al espíritu, tanto al que la ha creado como al que la juzga y disfruta" (1977, p. 88).

Empero, hemos de aclarar que con esto no se intenta llegar a una conclusión definitiva, sino solo hacer un acercamiento a dicha posibilidad. Así, pues, frente a la pregunta que plantea este tipo de arte -ies posible mantener la idea de que hay un tipo de comunicación conceptual en la relación entre la obra de arte y el observador? - hemos de recoger dos elementos generales del planteamiento de Kant: primero, es necesario hacer una dilucidación acerca de las características de la experiencia estética en cuanto a la posibilidad de juzgar una obra de arte, y, segundo, considerar

\footnotetext{
${ }^{1}$ Es necesario hacer una aclaración en este punto. El objetivo del texto no es mostrar una relación de identidad entre el 'concepto' que presenta el arte conceptual y las 'ideas estéticas' de Kant. El punto relevante y que a mi parecer resulta interesante radica en el modo de la comunicación de la idea/concepto. Aquello que hace posible la comunicación de la 'idea estética' puede darnos luces acerca de cómo podemos entender en el caso del arte conceptual el establecimiento de una posible comunicación entre la obra y el observador, vía un concepto. La pregunta central, por ende, es acerca de la posibilidad de la comunicación de ideas y esto en virtud de unas características formales de los términos de la relación artista-obra-observador, sin acudir por ello a realizar especulaciones en torno a la naturaleza de la materia conceptual. Dada esta caracterización de la pregunta, el presente texto asume la hipótesis según la cual podemos obviar una objeción de anacronismo en virtud de la permanencia y pertinencia de la pregunta, tanto para Kant como para el arte no figurativo.
} 
las condiciones y características de lo que podría reconocerse como una "idea estética" en la forma de la obra, cuestiones estas que encontramos en su Crítica del Juicio (1977).

\section{La Experiencia del Juicio Frente al a Obra de Arte}

¿Por qué hablamos aquí de 'experiencia del Juicio' y no de 'experiencia estética', término que usualmente asumimos como correlativo a la obra de arte? La respuesta es franca: frente a la sencillez de forma y materia de una obra como Hectáreas de heno y su pretendida posibilidad de expresión de un concepto, no es tan relevante hablar acerca de su estética en sentido tradicional, como un placer o emoción frente a la obra, como sí parece ser hacerlo acerca de una condición de la experiencia ante ella que permita captar la idea expresada. Esta condición, a mi modo de ver, se puede encontrar en lo que Kant llama la facultad de juzgar o Juicio, esto es, en la posibilidad de emitir un juicio valorativo frente al objeto en cuestión identificándolo como 'bello' -según la estética del siglo $\mathrm{XVIII}^{2}$-, pero antes que esto, como 'obra de arte' y, más aún, como 'expresión de una idea'. Lo que aquí denomino la 'experiencia del juicio' es expuesto por Kant en su Introducción a la tercera Crítica.

En general, Kant afirma que la facultad del espíritu del conocimiento de los individuos puede reducirse a tres: facultad de conocer, el sentimiento de placer y dolor, y la facultad de desear-lo cognitivo, lo estético y lo moral de la experiencia-. El sentimiento de placer se encuentra en una posición intermedia entre la facultad de conocer y la de desear, lo que equivale a decir que entre el entendimiento y la razón está el Juicio (facultad de juzgar). Y por esta peculiar condición instaura la posibilidad de realizar la comunicación necesaria, según un propio principio $a$ priori, entre la facultad de conocer y la facultad de desear.

El Juicio no es más que "la facultad de pensar lo particular como contenido en lo universal" (Kant, 1977); empero, Kant nos obliga a distinguir entre Juicio determinante y Juicio reflexionante. El primero corresponde al juicio de conocimiento: "Si lo universal (la regla, el principio, la ley) es dado, el Juicio, que subsume en él lo particular (incluso cuando como Juicio trascendental pone a priori las condiciones dentro de las cuales solamente puede subsumirse en lo general), es

${ }^{2}$ El juicio de lo bello puede ser considerado hoy en día como nada más que un juicio valorativo entre otros posibles. Lo cual no es ajeno a la postura kantiana, en la medida en que el 'juicio de lo feo' cabe dentro de la facultad reflexionante de juzgar. Lo interesante aquí es que aun para Kant el reconocimiento de un objeto como bello supone una aplicación del juicio - posiblemente no explícita- en torno al reconocimiento de un objeto como una obra de arte; de hecho en $\S^{51}$ Kant establece una relación directa entre juzgar una cosa como bella -siendo esta un producto del hombre- y reconocerla como obra de arte. Este juicio adquiere plena actualidad en la medida en que uno de los problemas centrales de la filosofía del arte contemporáneo -y de la filosofía del arte en general- es el reconocimiento de un objeto como obra de arte. 
determinante" (Kant, 1977). En la teoría kantiana, el Juicio determinante puede operar bajo las leyes universales trascendentales del entendimiento, esto es, las categorías, por lo cual no tiene necesidad de pensar una ley para subordinar lo particular en la naturaleza a lo universal. Por ello, el Juicio determinante es el que provee un conocimiento empírico de un objeto. En este Juicio la imaginación, como facultad de la sensibilidad, recorre el conjunto de intuiciones sensibles y las sintetiza de acuerdo con una regla provista por un concepto del entendimiento, de manera tal que, si cabe hablar de armonía entre estas facultades, diríamos, con Mark Johnson en Kant's unified theory of taste, que "aquí la imaginación está en armonía o concordancia con el entendimiento por la obvia razón de que la segunda facultad controla (mediante el concepto) la actividad de la primera facultad en tanto ésta unifica lo múltiple"(Johnson, 1979, p. 169).

Pero el Juicio reflexionante ocurre cuando solo lo particular es dado, y esta facultad debe encontrar lo universal, es decir, tiene que "ascender" de lo particular en la naturaleza a lo general y esto según un principio a priori, ya que este debe poder "fundar la unidad de todos los principios empíricos bajo principios igualmente empíricos, pero más altos, y así la posibilidad de la subordinación sistemática de los unos a los otros" (Kant, 1977, p. 106). Corresponde, por ende, al Juicio reflexionante darse a sí mismo como ley un principio trascendental.

El Juicio reflexionante puede, pues, tan solo darse a sí mismo, como ley, un principio semejante, trascendental, y no tomarlo de otra parte (pues entonces sería Juicio determinante) ni prescribirlo a la naturaleza, porque la reflexión sobre las leyes de la naturaleza se rige según la naturaleza, y esta no se rige según las condiciones según las cuales nosotros tratamos de adquirir de ella un concepto que, en relación a esas, es totalmente contingente (Kant, 1977, p. 106).

En el Juicio reflexionante no hay concepto determinado, por lo cual la armonía que es propia del Juicio entre la imaginación y el entendimiento no puede estar sujeta a las reglas del segundo; según Kant, en la reflexión las facultades se encuentran en un libre juego: en el Juicio reflexionante las facultades cognitivas entran en un libre juego frente al objeto por juzgar, aun si este no era el propósito del objeto -pues no hay concepto que determine la reflexión (la intención no es conocer el objeto)-. El libre juego de las facultades nace de la actividad reflexiva del Juicio, es decir, el darse a sí mismo un principio del Juicio genera la actividad de la armonía de las facultades, sin determinación de un concepto del entendimiento. Kant considera que el Juicio reflexionante es la forma de juicio que aplica a una obra de arte; de esta manera deviene juicio de gusto. 
Pero, ¿en qué consiste este juicio de gusto, cuál es su característica? Para responder a esta pregunta, primero es necesario aclarar cuál es el principio del Juicio reflexionante, a saber: "el principio del Juicio, con relación a la forma de las cosas de la naturaleza bajo leyes empíricas en general, es la finalidad de la naturaleza en su diversidad. Esto es, la naturaleza es representada mediante ese concepto, como si un entendimiento encerrase la base de la unidad de lo diverso de sus leyes empíricas" (Kant, 1977, p. 107). Si asumimos que las leyes empíricas pueden ser consideradas como si tuvieran necesidad según el entendimiento, entonces la reflexión en el Juicio consiste en ver la diversidad de la naturaleza como conforme a una finalidad. La finalidad es el principio a priori propio del Juicio reflexionante, pues no es que, de hecho, encuentre la finalidad de las cosas de la naturaleza, sino que propone esa finalidad para reflexionar sobre ella, para así subsumir bajo leyes, dadas por el Juicio reflexionante, la diversidad de los fenómenos. El principio de la finalidad formal de la naturaleza es el principio trascendental del Juicio.

Este principio trascendental responde entonces al problema de cómo es posible a partir de las percepciones de la naturaleza, que guardan una "infinita diversidad de leyes empíricas", realizar una "experiencia coherente". La experiencia, en cuanto el conocimiento, está limitada por las leyes generales por las cuales es posible pensarla, ya que descansan en las categorías; bajo esas leyes el Juicio es determinante. Pero los objetos de la naturaleza son determinables de otros diversos modos, aunque contingentes (un objeto puede ser inicio de muchas cadenas causales según sus propiedades empíricas). Sin embargo, la unidad de la experiencia debe ser pensada con necesidad, para comprender la conexión general de los conocimientos empíricos con el todo de la experiencia; afirmar la posibilidad de esta unidad de lo diverso es la función del Juicio reflexionante.

Para Kant el entendimiento no puede determinar nada en los objetos empíricos, pero, para buscar las leyes empíricas, puede poner en la reflexión sobre las mismas el principio a priori de la finalidad, es decir, "que una ordenación cognoscible de la naturaleza es posible según ellas" (1977, p. 112). La finalidad apunta, entonces, a la representación de esa concordancia necesaria entre entendimiento y naturaleza como condición de posibilidad de conocimiento en la experiencia empírica. "Esa concordancia de la naturaleza con nuestra facultad de conocimiento es presupuesta a priori por el Juicio para su reflexión sobre aquella según sus leyes empíricas, reconociéndola el entendimiento como objetiva y contingente a un mismo tiempo y atribuyéndola solo el Juicio a la naturaleza como finalidad trascendental" (Kant, 1977, p. 112). El Juicio afirma este principio a priori que, empero, solo es viable como una prescripción de la ley por parte del sujeto y para sí mismo, para la reflexión sobre la naturaleza. 
Ahora bien, ¿por qué considera Kant que en el Juicio reflexionante radica la posibilidad de juzgar una obra de arte? Una obra de arte es también una cosa en el mundo, luego, de ella tenemos intuiciones sensibles y podemos conocerla en tanto fenómeno; sin embargo, Kant considera que hay un plus en la experiencia de la obra que no se limita a la facultad de conocer. En el momento de juzgar una obra experimentamos un particular sentimiento de placer o dolor. Pero Kant no está afirmando aquí una teoría emotivista del arte; todo lo contrario, este sentimiento es propio de la posibilidad de pensar la concordancia de la naturaleza con "nuestra intención" de conocerla; es un sentimiento determinado por un principio a priori "valedero para cada cual, y es, a saber, tan solo la relación del objeto con la facultad de conocer, sin que el concepto de la finalidad se refiera aquí en lo más mínimo a la facultad de desear, diferenciándose así completamente de toda finalidad práctica de la naturaleza" (1977, p. 115).

El sentimiento de placer que nace del principio de finalidad sobreviene en la posibilidad de descubrir la coherencia de la naturaleza que encontramos en nuestro esfuerzo por conocerla. El placer del que habla Kant está ligado a la posibilidad de sentir un crecimiento de nuestro conocimiento. Solo de esta manera podemos hablar de una 'experiencia estética', cuyo objeto privilegiado no es particularmente el arte, sino la naturaleza. Así, pues, si la obra de arte despierta dicho sentimiento, hemos de asumir, según Kant, que la obra misma comparte esta experiencia solo en la medida en que parece naturaleza, es decir, que en un juicio de gusto la representación del objeto parece exhibir finalidad para mis facultades aunque no haya de hecho un fin definido ${ }^{3}$.

La "cualidad estética" de la representación emerge de la posibilidad de ligar a esta la finalidad de la naturaleza. Sin embargo, el sentimiento de placer o dolor que va unido a la representación es lo subjetivo que no puede ser nunca un elemento de conocimiento, "aunque él pueda ser el efecto de algún conocimiento". Por ello, la finalidad de la representación de una cosa es lo subjetivo mismo que precede al conocimiento de un objeto, tal que el objeto es siempre 'final' o 'conforme a fines' "solo porque su representación está inmediatamente unida con el sentimiento del placer, y esta representación misma es una representación estética de la finalidad" (Kant, 1977, p. 118).

Así, el placer del que habla Kant es aquel que va unido a la simple aprehensión de la forma de un objeto y que expresa su concordancia con las facultades de conocer que están en juego en el Juicio reflexionante: es el placer que nace de la subjetiva y

\footnotetext{
3 "En un producto del arte bello hay que tomar consciencia de que es arte y no naturaleza; sin embargo, la finalidad en la forma del mismo debe parecer tan libre de toda violencia de reglas caprichosas como si fuera un producto de la mera naturaleza" (Kant, 1977, p. 261). Ver también: Johnson(1979).
} 
formal finalidad del objeto. Pero no hay que confundir el juicio de gusto, que corresponde al sentimiento de placer, con un juicio de lo agradable; tanto el sentimiento de placer kantiano como lo agradable son estados subjetivos, pero lo agradable no puede ser compartido por nadie, mientras que, para Kant, el sentimiento en el juicio de gusto siempre espera una validez universal. En la medida en que el sentimiento es, valga la expresión, un 'estado mental' de las facultades de conocer que nace de su armonía en el Juicio, es posible esperar que el juicio obtenga validez universal para todo ser racional. Y esto es resultado de hallar la finalidad de la forma del objeto de gusto, para nuestro caso, la obra de arte. Johnson (1979) muestra que Kant comprende 'forma' como la estructura, relación u orden de elementos ${ }^{4}$. La estructura es algo que excede lo agradable de la representación del objeto y que no está sujeto a las contingencias de la percepción (puede que no todos podamos ver de la misma manera una mancha de color); por ello, "solo la forma puede suplir la clase peculiar de validez universal atribuida al juicio de gusto" (Johnson, 1979, p. 170). Es por ello, según Kant, que al arte le compete el juicio de gusto, pues en su forma, su composición, encontramos finalidad en el momento del juicio, $y$, además, en virtud de esta podemos exigir validez universal para él, aun si es cierto que el juicio de gusto expresa nuestro sentimiento de placer -el cual es siempre subjetivo-. El arte, entonces, produce formas con finalidad ${ }^{5}$.

En conclusión, cuando la imaginación (facultad de intuiciones a priori) entra en relación con el entendimiento (facultad de los conceptos) por medio de una representación y ello produce un sentimiento de placer, entonces el Juicio reflexionante considera el objeto como conforme a la finalidad, y el juicio que se realiza en este momento es un juicio estético sobre la finalidad del objeto y, esto, según su forma. En efecto, el Juicio reflexionante atiende a la forma del objeto (no lo material de su representación como sensación) "como la base de un placer en la representación de semejante objeto, con cuya representación este placer es juzgado como necesariamente unido, y consiguientemente, no solo para el sujeto que aprehende aquella forma, sino para todo el que juzga en general" (Kant, 1977, p. 119). A partir de esta experiencia fundada en el Juicio reflexionante este objeto puede llamarse 'bello', y la facultad de emitir juicios según dicho placer -con una cierta pretensión de universalidad- es lo que Kant identifica como 'gusto'. El

\footnotetext{
${ }^{4} \mathrm{Al}$ respecto, afirma: "Form in a beautiful object, then, will consist of either (1) relation of objects (shapes, figures) and relation of parts (inner structure) in space, as in painting, sculpture, landscape, gardening, etc., or $\left(^{2}\right)$ relation of sensations in time, as in music, poetry, film, drama" (Johnson, 1979, p. 170). prichosas como si fuera un producto de la mera naturaleza" (Kant, 1977, p. 261). Ver también: Johnson(1979).

${ }^{5} \mathrm{Al}$ respecto, Paul Guyer afirma: "In claiming that fine art is "purposive on its own", Kant is claiming that it (unlike merely agreeable art) produces purposive forms (material for reflection); and in stating that the enhancement of our capacity for social communication is attained "without a purpose", he seems to be suggesting that this is the effect of the art" (Guyer, 1997, p. 274).
} 
fundamento del placer se encuentra solo en la forma del objeto para la reflexión en general, no en la sensación ni en una relación con un concepto; y el gusto nace de las condiciones a priori, por ende, con valor universal de la representación del objeto en la reflexión. Lo particular del juicio de gusto, lo que lo diferencia de los demás juicios empíricos, es que es un sentimiento de placer -no un concepto- lo que se exige a cada uno en la medida en que puede estar ligado a la representación del objeto de juicio. Así, pues, a partir del placer que el sujeto experimenta en la reflexión emite un juicio empírico e individual, un juicio de gusto que pretende la aprobación de cada uno en la medida en que "la base de este placer se encuentra en la condición universal, aunque subjetiva, de los juicios reflexionantes, que es, a saber: la concordancia final de un objeto (sea producto de la naturaleza o del arte) con la relación de las facultades de conocer entre sí, exigidas para todo conocimiento empírico (la imaginación y el entendimiento)"(Kant, 1977, p. 120).

\section{Hacia la Comunicación de Ideas Estéticas}

Expuesto lo que significa la reflexión en la facultad de juzgar y su pertinencia para la experiencia de la obra de arte, hemos de pasar ahora al punto central: ¿Es posible la comunicación de ideas en la relación entre la obra y el observador? Para responder a esta pregunta es necesario comprender primero los términos de la relación. Dadas las diferencias enunciadas entre Juicio reflexionante y Juicio determinante, es claro que si una regla o concepto del entendimiento estuviera ligada a la producción de la obra, tendríamos que afirmar, de acuerdo con Kant, que la imaginación del artista perdería su libertad creativa, es decir, no podría entrar a jugar libremente; lo mismo ocurriría para el observador, pues él también sería constreñido por el concepto previniendo así el libre juego de sus facultades en la reflexión y, con ello, el correspondiente sentimiento de placer frente a la obra. Es así como, en palabras de Allison, en Kant's theory of taste, podemos afirmar que "hay una importante correlación entre la libre armonía de las facultades requerida para la creación del arte y aquella que es requerida para su deleite" (2001, p. 281).

Podemos entonces identificar el gusto como el plano general de la experiencia y la comunicación de ideas de la obra de arte; pero, además, la estética kantiana pone de relieve un término que adquiere mayor importancia que el observador: el artista, identificado con el concepto de genio. El artista es el encargado de producir la forma $\mathrm{y}$, en la medida en que esta concuerda con el gusto, la finalidad de la misma. Pero la producción de la forma se revela, según Kant, como expresión de conceptos -no su determinación para el entendimiento- que, en virtud de ella, pueden ser universalmente comunicables. Además, en tanto la imaginación del artista se 
encuentra libre de las determinaciones de los conceptos del entendimiento, estos conceptos no están limitados a la experiencia fenoménica, sino que son más semejantes a las ideas de la razón que remiten a cosas en sí, a lo suprasensible o inefable, pero para hacerlas universalmente comunicables en la obra sin imposición de reglas. Sin embargo, no son ideas de la razón (Kant desplaza la libre armonía entre imaginación y razón para el sentimiento de lo sublime), pues la obra de arte es un producto $y$, ante todo, una materia sensible; por ende, para nuestro entendimiento es siempre fenómeno, y la idea expresada por el artista debe poder ser representable -aunque no determinada por una regla-y en esa medida excede la mera experiencia empírica ${ }^{6}$. De ahí que la respuesta a la pregunta requiere una anterior condición en el acto creativo dictado por la capacidad del artista y la expresión de las ideas. Es por ello que la imaginación del genio puede proporcionar:

Una materia no desarrollada y abundante para el entendimiento, a la cual este, en sus conceptos, no puso atención [...] resulta que el genio consiste propiamente en la proporción feliz, que ninguna ciencia puede enseñar y ninguna laboriosidad aprender, para encontrar ideas a un concepto dado, y dar, por otra parte, con la expresión mediante la cual la disposición subjetiva del espíritu producida pueda ser comunicada a otros como acompañamiento de un concepto (Kant, 1977, p. 274).

En la medida en que el 'arte bello' debe parecer naturaleza -esto es, estar libre de restricción de reglas- y que el arte es producto del genio -el artista-, Kant afirma que el genio es un talento natural por medio del cual la naturaleza da la regla al arte. Lo cual quiere decir, en palabras de Gadamer, que "para lo bello en el arte no existe tampoco ningún otro principio de enjuiciamiento, ningún otro patrón del concepto y del conocimiento que el de la idoneidad para el sentimiento de la libertad en el juego

\footnotetext{
${ }^{6}$ Vale la pena hacer una breve distinción entre los conceptos de entendimiento, ideas estéticas e ideas de la razón. Los conceptos del entendimiento son conceptos de la naturaleza por medio de los cuales el entendimiento provee un conocimiento teórico de la naturaleza, mientras que las ideas de la razón son conceptos de la libertad cuya legislación la realiza la razón y es práctica; los conceptos de la naturaleza representan las cosas como meros fenómenos, y el concepto de libertad "representa en sus objetos una cosa en sí misma, pero no lo hace en la intuición, y por lo tanto ninguno de los dos puede producir un conocimiento teórico de su objeto como cosa en sí" (Kant, 1977, p. 100). Esto supone que las ideas de la razón remiten a lo suprasensible, un campo inaccesible para la facultad de conocer; por lo cual estas ideas no pueden tener más que una realidad práctica. El Juicio tiene su esfera en la mediación entre las dos anteriores, en la medida en que subsume lo indeterminado en la naturaleza, en la forma de la finalidad, es decir, la legislación del concepto de libertad: "el concepto de libertad debe realizar en el mundo sensible el fin propuesto por sus leyes, y la naturaleza, por tanto, debe poder pensarse de tal modo que al menos la legalidad de su forma concuerde con la posibilidad de los fines, según leyes de libertad, que se han de realizar en ella" (p. 101). Las ideas estéticas participan de esta mediación en la medida en que hacen sensible, mediante la forma de la obra de arte (producto del artista), un elemento indeterminado que puede ser pensado con finalidad y este es un principio práctico de la razón que funciona como idea regulativa para la reflexión en el Juicio.
} 
de nuestra capacidad de conocer" (Gadamer, 1977, p. 90). Genio es la capacidad de producir una obra que tenga finalidad para nuestras facultades; diríase del artista con este talento que tiene una disposición natural al libre juego de las facultades y, con ello, a incitarlo en el observador. En términos de Kant, "las ideas del artista despiertan ideas semejantes en su discípulo cuando la naturaleza lo ha provisto de una proporción semejante de las facultades del espíritu" (Kant, 1997, p. 256). Pero no podemos asumir que el observador sea un discípulo en potencia, puede que por el contrario no tenga talento alguno para el arte. Y sin embargo, podemos esperar que frente a la obra del genio tenga una cierta experiencia. Lo que esperamos con Kant es que su experiencia sea tal que tenga 'ideas semejantes' al menos en su reflexión frente a la obra. En esto radica la posibilidad de hablar de una experiencia de comunicación de ideas: "el arte del genio consiste en hacer comunicable el libre juego de las fuerzas del conocimiento. Esto es lo que hacen las ideas estéticas que él inventa" (Gadamer, 1977, p. 88).

Las ideas estéticas están relacionadas con la capacidad productiva de la imaginación, que Kant comprende como el poder de crear otra naturaleza a partir del material que la naturaleza provee. Esto es posible en la medida en que procesa este material -los datos de la intuición sensible- en algo que sobrepasa la naturaleza. La obra de arte se compone de ciertos "atributos estéticos" que constituyen la materia de una idea estética y que, en conjunto, dan lugar a su forma 0 estructura, y es en virtud de esta que la idea estética se hace universalmente comunicable (Cfr., Allison, 2001, p. 283). Esta composición resultante es la que debe expresar finalidad en la forma, tal que produzca el sentimiento de placer en el Juicio. Es así, por ende, que una idea estética consiste en la "representación de la imaginación que incita a pensar mucho, sin que, sin embargo, pueda serle adecuado pensamiento alguno, es decir, concepto alguno, y que, por lo tanto, ningún lenguaje expresa del todo ni puede hacer comprensible" (Kant, 1977, p. 270). Las ideas estéticas tienden a algo por encima de los límites de la experiencia, son ideas 'emparentadas', si se me permite la expresión, con ideas de la razón; la distinción con estas radica en su vocación a hacer sensible la idea, aunque indirectamente. Ideas tales como el 'paraíso', el 'infierno', el 'mal', la 'eternidad', la 'finitud', la 'banalidad', los 'vicios', el 'amor', son el tipo de ideas que hacen comunicable el arte en una forma sensible que supera las barreras de la experiencia e incita el libre juego de la imaginación.

\footnotetext{
${ }^{7}$ Kant define "atributos estéticos" de la siguiente manera: "Las formas que no constituyen la exposición de un concepto dado, sino solo expresan, como representaciones adyacentes de la imaginación, las consecuencias allí enlazadas y el parentesco con otras, llámanse atributos (estéticos) de un objeto cuyo concepto, como idea de la razón, no puede ser expuesto adecuadamente"(1977, p. 272).
} 
Con esta característica de la idea estética se abre la posibilidad de hablar de una comunicación de ideas en la experiencia de la obra de arte. No es que todos, frente a la representación del objeto (las bolsas llenas de heno), tengamos la misma idea; la idea no es transparente como si fuera una proposición (un juicio determinante). Aunque todos tengamos, por ejemplo, la idea de la 'banalidad de la riqueza' que podríamos decir expresa la obra de Salcedo, la multiplicidad de pensamientos que incita la obra -y con ello el sentimiento de placer en el Juicio-no puede ser recogido en un mismo concepto para todos ${ }^{8}$. Es por ello que Kant afirma que en la medida en que la idea estética "ocasiona tanto pensamiento que no se deja nunca recoger en un determinado concepto, y, por tanto, extiende estéticamente el concepto mismo de un modo ilimitado, entonces la imaginación, en esto, es creadora y pone en movimiento la facultad de ideas intelectuales para pensar, en ocasión de una representación (cosa que pertenece ciertamente al concepto del objeto), más de lo que puede en ella ser aprehendido y aclarado" (1977, p. 272). A la expresión de ideas estéticas le corresponde el margen de lo indeterminado por el entendimiento, de lo inefable como expresión y, por ende, de una indirecta relación con lo suprasensible como idea de la razón.

En consecuencia, nuestra cuestión sobre la posibilidad de comunicación de ideas en la experiencia de la obra de arte sigue la doble condición del juicio de gusto, pues, por una parte, la idea estética es universalmente comunicable en virtud del genio y la finalidad de la forma en la obra que la hacen un objeto conforme al gusto; pero, por otra parte, la idea estética no puede ser determinada, por ende, no puede ser objetivamente compartida, sino que mantiene siempre el sustrato subjetivo del sentimiento de placer que nace de la multiplicidad de pensamientos que la idea estética incita en el Juicio reflexionante y que es, en principio, inagotable. Es bajo esta duplicidad del Juicio que podemos hablar en arte de una comunicación de ideas.

Pero no hemos de confundir juicio estético conforme al gusto e idea estética. Al

\footnotetext{
${ }^{8}$ Johnson propone otro ejemplo: "let us take, for example, a poet who wishes to express his rational idea of the waning of faith in the modern world. There can be no adequate intuitive presentation of this idea, because there are no marks in any intuition adequate to a rational idea. But the poet's genius supplies him with anaesthetical idea (e.g., the sea of faith, as in M. Arnold's "Dover Beach"), which may be associated with the rational idea and which takes the place of the unavailable logical (intuitive) presentation. [...] the metaphor of the sea suggest a wealth of thought applicable to faith -the sea is a source of life, its unfathomable depths suggest inscrutable mystery, it both unites and separates persons of all cultures, nations and historical periodsthese and many other thoughts serve, in the context of the poem, to express the poet's conception of the reality of faith. The aesthetical idea is both occasioned by (in the poet) and occasions (in the hearer) a free play of the cognitive faculties for which no concept is adequate. As I experience the poem, my reflection upon its forms (aesthetical attributes) constitutes an harmonious interplay of my imagination and understanding which is yet a free accordance" (Johnson, 1979, p. 173).
} 
juicio estético corresponde el plano del observador; mientras que a la idea estética, el de la forma. Lo que permite la comunicación de la idea estética es la forma como el genio expresa en su obra. El juicio estético ocurre cuando esta idea entra en el momento del juicio, es decir, pone a reflexionar la imaginación y el entendimiento del observador. Claramente, como habíamos anunciado, el libre juego de las facultades es necesario en el genio y en el observador, pero las funciones son distintas: en el primero es creativo, y la comunicación se establece en la forma; en el segundo, el juicio despierta el sentimiento de placer propio del Juicio reflexionante y se establece lo que llamo la duplicidad del Juicio; pero esta doble condición del Juicio es propia de la posibilidad de exigir una validez universal al juicio estético, y esto en virtud del sensus communis, esto es, de la posibilidad de comparar los juicios (posibles) de otros "atendiendo tan solo a las características formales de la propia representación o del propio estado de representación" (Kant, 1977, p. 245). Por ello, la idea estética no puede ser determinada objetivamente, pues lo que se comparte es la posición, en tanto participamos de la misma facultad del gusto, un sensus communis en virtud del cual exigimos la universal comunicabilidad del sentimiento $\mathrm{y}$, sin embargo, la multitud de pensamientos no puede ser determinada por concepto alguno, guardando el margen de subjetividad en la materia de la representación.

La forma de la idea estética sirve, entonces, de exposición lógica al concepto, aunque la obra siempre abriría "la perspectiva de un campo inmenso de representaciones afines" para el observador que, ante esta multiplicidad, no puede esperar sino que su sentimiento de placer sea compartido por sus iguales. "En una palabra, la idea estética es una representación de la imaginación emparejada a un concepto dado y unida con tal diversidad de representaciones parciales en el uso libre de la misma, que no se puede para ella encontrar una expresión que indique un determinado concepto; hace pues que en un concepto pensemos muchas cosas inefables, cuyo sentimiento vivifica las facultades de conocer" (Kant, 1977, p. 274). El arte, bajo esta perspectiva, es, por tanto, expresión de conceptos que son comunicados más allá de sus cualidades sensibles (en el caso de Salcedo, plástico y heno); su forma y atributos (bolsas amontonadas y marcadas) son ya, ellas mismas, expresión de la idea estética, del concepto mismo que el artista ha puesto en la obra. La experiencia de ser incitado por la obra a pensar libremente, aunque sea subjetiva en su contenido, es el sentido que podemos dar a la exigencia del arte por establecer una comunicación conceptual con el objeto. 


\section{Referencias}

Allison, H. (2001). Kant's theory of taste. Cambridge: Cambridge University Press. Gadamer, H.G. (1977). Verdad y método. Salamanca: Sígueme.

Guyer, P. (1997). Kant and the claims of taste. Cambridge: Cambridge University Press.

Johnson, M. (Winter 1979). "Kant's unified theory of taste". Journal of aesthetics and art criticism, Vol. 38, No. 2.

Kant, I. (1977). Crítica del Juicio. Madrid: Espasa Calpe. 